Service social

\title{
La dynamique traumatisante des abus sexuels et leurs conséquences à long terme
}

\section{Dominique Damant}

Volume 42, numéro 2, 1993

Recherche et pensée critique

URI : https://id.erudit.org/iderudit/706617ar

DOI : https://doi.org/10.7202/706617ar

Aller au sommaire du numéro

Éditeur(s)

École de service social de l'Université Laval

ISSN

1708-1734 (numérique)

Découvrir la revue

Citer cet article

Damant, D. (1993). La dynamique traumatisante des abus sexuels et leurs conséquences à long terme. Service social, 42(2), 51-61.

https://doi.org/10.7202/706617ar
Résumé de l'article

Cet article fait une revue des écrits portant sur les conséquences à long terme des abus sexuels et de l'inceste. L/auteure présente la conceptualisation des quatre dynamiques traumatisantes de Browne et Finkelhor. Ce modèle a l'avantage d'organiser la liste des innombrables conséquences en un tout cohérent. Après avoir décrit l'apport de cette conceptualisation à l'étude des abus sexuels et de leurs conséquences, l'auteure souligne ses limites à l'aide de la théorie de l'attribution et plus particulièrement de la théorie féministe. 


\title{
La dynamique traumatisante des abus sexuels et leurs conséquences à long terme
}

\author{
Dominique DAMANT \\ Chargée de cours \\ et étudiante au doctorat en service social \\ Université Laval
}

\section{INTRODUCTION}

Pendant longtemps, les cliniciens et même les chercheurs ont ignoré l'existence des abus sexuels et de l'inceste. Aujourd'hui, plus personne ne nie cette réalité. Toutefois, on trouve plusieurs termes dans la littérature pour décrire un contact sexuel entre un adulte et un enfant: abus sexuel, molestation, victimisation sexuelle, exploitation sexuelle, assaut sexuel. Le terme abus sexuel nous révèle sa filiation avec les abus faits aux enfants, alors que d'autres termes comme molestation, victimisation ou exploitation reflètent les liens avec d'autres formes de violence faites aux femmes.

Une certaine confusion existe encore sur le plan de la terminologie. Les abus sexuels font généralement référence à un contact sexuel entre un adulte et un enfant, alors qu'un inceste représente des gestes sexuels entre des personnes apparentées. Tous les incestes ne sont pas des abus sexuels et tous les abus sexuels ne sont pas incestueux. Un inceste père-fille mineure est donc un abus sexuel, alors que des jeux sexuels entre frères et soeurs qui ont environ le même âge sont incestueux, mais pas nécessairement abusifs. 
Jusqu'au début des années 70, certains auteurs ont continué à prétendre que l'inceste et les abus sexuels pouvaient ne pas avoir d'effets nocifs sur la victime (Bender et Balu, 1937; Sloane et Karpinski, 1942 ; Bender et Grugett, 1952 et Lukianowicz, 1972, tous cités par Conte, 1985). Toutefois, ces études sont des récits anecdotiques d'expériences cliniques et contiennent plusieurs biais (Conte, 1985). Dès 1969, De Francis (De Francis, 1969, cité par Finkelhor, 1984) reconnaissait que $66 \%$ des victimes interrogées étaient perturbées par l'abus.

À partir des années 70, les auteurs reconnaissent l'existence de conséquences des abus sexuels et se demandent plutôt quel type de séquelles ces derniers peuvent laisser. Les études de cette époque contiennent encore pour la plupart de nombreux écueils méthodologiques. Les échantillons sont petits; souvent, on n'en parle même pas. Ce sont, de façon majoritaire, des échantillons cliniques. Les outils de mesure et la procédure ne sont pas décrits ou le sont de façon confuse ou partielle. On y trouve peu d'analyses statistiques des données. À ce stade des recherches sur les conséquences des abus sexuels, il était quasi impossible de faire des corrélations spécifiques entre l'inceste et les abus sexuels et certains problèmes vécus par les victimes.

Plusieurs études sérieuses, dont trois avec des échantillons probabilistes pris dans la communauté (Bagley et Ramsey, 1986; Peters, 1984; Russell, 1984), ont trouvé que les victimes d'abus sexuels dans la communauté ressentaient des effets durables à divers degrés. C'est dire que, non seulement les populations cliniques sont affectées par les abus sexuels, mais que même les victimes qui ne consultent pas peuvent avoir des séquelles.

Il est possible de regrouper ces problèmes sous différents aspects : émotionnel, cognitif, somatique, comportemental et interpersonnel. Plus particulièrement, Finkelhor et Browne (1985) ont décrit quatre dynamiques traumatisantes propres aux abus sexuels à partir desquelles les différentes conséquences peuvent être regroupées. Ces dynamiques permettent de saisir le processus qui cause le traumatisme et les séquelles qu'il entraîne. Le but du présent article est donc de faire une recension des écrits sur les conséquences à long terme des abus sexuels sous l'angle du modèle de Finkelhor et Browne.

\section{LES QUATRE D YNAMIQUES TRAUMATISANTES}

Selon Finkelhor et Browne, une dynamique traumatisante est une expérience qui modifie la perception cognitive ou émotive du monde et qui cause un traumatisme en faussant le concept de soi de l'enfant, sa vision du monde et ses capacités affectives. En ce qui concerne les abus sexuels, il existe quatre 
dynamiques traumatisantes: la sexualisation traumatisante, la stigmatisation, la trahison et l'impuissance. C'est à partir de ces quatre dynamiques que l'on peut situer le processus des abus sexuels tant pour les enfants que pour les adultes et que l'on peut définir leurs conséquences.

Chaque abus sexuel est différent, car on peut y retrouver en dominance une dynamique particulière. C'est toutefois la conjonction des quatre éléments qui distingue les abus sexuels des autres formes d'abus. À chacune de ces dynamiques se greffent un ensemble de réactions immédiates et de conséquences à long terme, sur le plan de l'impact psychologique et des manifestations comportementales. Regardons de plus près chacune des ces quatre dynamiques.

\section{La sexualisation traumatisante}

Les auteurs définissent la sexualisation traumatisante comme un processus par lequel, à la suite de l'abus sexuel, la sexualité d'un enfant est moulée de façon inappropriée dans son développement et de façon dysfonctionnelle dans ses relations interpersonnelles. En d'autres mots, l'enfant participe à des activités sexuelles qui ne sont pas conformes à son stade de développement et qui s'inscrivent dans une relation où la sexualité ne devrait pas s'actualiser.

Le traumatisme s'explique par les éléments suivants de l'expérience d'abus. Tout d'abord, les activités sexuelles qui se vivent au cours de tout abus sexuel - qu'il soit incestueux ou non - sont des événements que la plupart des enfants de l'âge des victimes ne connaissent pas. Par ailleurs, comme le tabou de l'inceste est quasi universel et que les activités entre parents sont interdites par ce tabou, sa transgression pervertit la relation parent-enfant.

Par ailleurs, les relations sexuelles entre adultes et enfants posent toute la question du consentement. Un enfant peut-il consentir librement à quelque chose qu'il ne connaît pas pleinement? Nous souscrivons ici à la thèse de Herman (1981), selon laquelle le consentement ne peut exister dans des relations sexuelles entre adultes et enfants. Les enfants ne sont pas libres de $s^{\prime}$ engager dans des relations sexuelles avec des adultes. C'est particulièrement vrai dans les rapports entre les enfants et leurs parents, alors que les enfants dépendent de l'adulte pour leur survie matérielle et affective.

Le degré auquel un abus est sexualisé peut varier. Quand l'abuseur cherche à provoquer une réponse sexuelle chez l'enfant ou qu'il incite l'enfant à participer activement à l'activité, la relation peut s'avérer plus traumatisante pour ce dernier. L'enfant se sent plus engagé dans la relation s'il ressent des sensations physiques. Cela peut déclencher un sentiment de confusion et de culpabilité. Il acquiert par le fait même des conceptions confuses au sujet des comportements sexuels appropriés et de la morale sexuelle (Finkelhor, 1986). 
Enfin, les enfants victimes d'abus sexuels sont souvent récompensés pour des comportements sexuels inappropriés à leur niveau de développement. L'enfant reçoit des cadeaux ou des privilèges par rapport aux autres enfants, quand l'abus se produit à l'intérieur de la famille. Et ces récompenses amènent les enfants à utiliser leurs comportements sexuels comme stratégie de manipulation pour répondre à leurs besoins.

Comme conséquence immédiate, il n'est pas étonnant de remarquer que ces enfants ont des préoccupations sexuelles inappropriées pour leur âge et des comportements sexuels compulsifs, de même que de la curiosité sexuelle excessive, voire compulsive (Finkelhor, 1986).

De plus, le concept de soi sexuel peut être affecté par cette confusion. Les enfants ont peu de capacité d'abstraction. Ils associent souvent leur concept de soi à des parties particulières de leur corps, à l'exclusion d'autres parties (Coopersmith, 1967). L'abus centrera l'attention de l'enfant sur ces parties génitales et il aura tendance à généraliser à l'ensemble de sa personne les messages qu'on lui aura ainsi transmis.

La sexualisation traumatisante a aussi des répercussions à l'âge adulte. Certains auteurs affirment que certaines victimes ont peur de la sexualité (Gorcey et al., 1986; Becker et al., 1984; Briere et Runtz,1987), alors que d'autres développent un dégoût, une aversion, de l'anxiété ou encore de la culpabilité à son sujet (Bagley et MacDonald, 1984; Jehu, 1989; Herman et al., 1986; Russell, 1986).

Par conséquence, les femmes victimes d'abus étant enfants ressentent peu de désir et manifestent des dysfonctions sur le plan du désir et de l'excitabilité (Becker et al., 1984 ; Tsai, 1979).

À l'autre extrémité de la balance, certaines victimes adoptent un comportement de promiscuité: elles changent souvent de partenaires sexuels (Tsai et al., 1979) ou elles vivent une sexualité compulsive (Meiselman, 1978; Herman, 1981 ; Courtois, 1979) allant jusqu'à la prostitution (Earls et David, 1990; James et Mayerling, 1979; Silbert et Pines, 1981, cités par Finkelhor, 1986).

\section{La stigmatisation}

Un stigmate est une cicatrice, une marque laissée par une blessure. Par extension, un stigmate psychologique est la cicatrice d'une blessure laissée sur le plan émotif. La stigmatisation est donc le mécanisme qui transmet à l'enfant des messages négatifs à son égard, modifiant par le fait même son image de soi.

Ce processus s'opère de différentes façons. Tout d'abord, l'abuseur et parfois même l'entourage de la victime peuvent blâmer et dénigrer la victime et la considérer comme un bien avarié. L'abuseur exerce également de la 
pression pour imposer le silence à l'enfant, silence qui provoque des sentiments de culpabilité et de honte. En effet, I'enfant qui se tait, pour se protéger de la réaction de l'entourage, pour protéger l'abuseur ou se protéger de celui-ci, se sent lié à ce dernier. Il s'ensuit que la victime se sent responsable de l'abus et développe donc de la honte et de la culpabilité au sujet des activités abusives.

La réaction de l'entourage peut également induire des sentiments de honte et de culpabilité. On sait qu'un enfant a tendance à penser qu'un adulte, et surtout un parent, a toujours raison. Quand I'abus est dévoilé, I'entourage de l'enfant peut le tenir responsable des événements. Les proches qui ne croient pas l'enfant ou qui l'accusent d'avoir provoqué l'abuseur exacerbent ainsi les sentiments de responsabilité et de culpabilité de la victime.

La connaissance des tabous sociaux à l'égard de l'inceste peut également augmenter sa culpabilité. Sachant que les événements qu'elle vit sont interdits socialement, la victime se sent encore plus coupable et méchante.

Les conséquences de la stigmatisation sont nombreuses. Ces messages amènent l'enfant à se sentir méchant, honteux et coupable. Tout d'abord, la victime qui se juge mauvaise s'isole de ses pairs. Les familles elles-mêmes, où se produisent des abus sexuels, sont souvent isolées socialement (Finkelhor, 1979). L'abus ne fait donc qu'augmenter l'isolement de l'enfant. La victime pourra aussi s'associer avec des pairs marginaux, en adoptant des comportements antisociaux de délinquance (Finkelhor, 1986). On remarque également une mauvaise estime de soi chez les jeunes victimes. Celles-ci peuvent aussi adopter des comportements suicidaires (De Young, 1982).

Les conséquences de la stigmatisation se manifestent aussi à long terme. Ainsi, les séquelles de la stigmatisation sur l'image de soi que développe la victime sont importantes et l'estime de soi s'en trouve affectée. En effet, plusieurs études, tant avec un échantillon clinique (Briere, 1984; Herman, 1981) qu'avec des populations non cliniques (Bagley et Ramsey, 1985 ; Courtois, 1979 ; Russell, 1986), parlent de faible estime de soi. Ces séquelles sont dévastatrices, tant sur le développement de la personne que sur ses capacités personnelles et interpersonnelles.

Les sentiments de culpabilité amènent la victime à se sentir responsable de l'abus. Nous parlons alors d'auto-responsabilisation (Hoagwood, 1990).

La stigmatisation contribue également à produire des sentiments dépressifs. En effet, les sentiments de honte et de culpabilité provoqués par la stigmatisation peuvent susciter à long terme de la dépression. Ainsi, plusieurs recherches avec des populations cliniques (Meiselman, 1978; Herman, 1981 ; Hartman, Finn et Leon, 1987 ; Gorcey, Santiago et McCall-Perez,1986; Wheeler et Walton, 1987) et d'autres avec des populations prises dans la communauté (Bagley et Ramsey,1985; Sedney et Brooks, 1984 ; Briere et 
Runtz,1985 ; Peters, 1984 ; Russell, 1986), ont trouvé que les victimes d'abus souffraient de dépression. Dans certains cas, cette tendance va jusqu'aux comportements suicidaires (Sedney et Brooks, 1984; Herman, 1981 ; Briere, 1984; Hartman, Finn et Leon, 1987).

Penchons-nous maintenant sur le processus de trahison.

\section{La trahison}

La trahison est le processus par lequel une personne dont dépend la victime lui cause du tort (Finkelhor et Browne, 1985). Au moment de l'abus, l'enfant réalise qu'une personne, en qui elle a confiance, la trahit et ne respecte pas ses besoins. Elle peut également réaliser plus tard qu'on l'a trompée en lui mentant. Et, plus l'enfant a un rapport de confiance avec l'abuseur, plus I'incident sera traumatisant.

Le sentiment de trahison est également vécu par rapport à d'autres membres de la famille, qui n'ont pu empêcher l'abus, qui n'ont pas cru l'enfant ou qui ne lui ont pas montré comment se protéger. L'absence de la mère (absence réelle, absence émotive ou incapacité à protéger sa fille) dans les cas d'abus sexuels est fortement documentée (Herman, 1981 ; Finkelhor, 1979).

L'attitude de l'entourage, au moment du dévoilement de l'abus, intervient également ici. Croit-on la victime, la blâme-t-on? Est-elle placée en dehors de la famille? Ainsi la position, non seulement de la famille immédiate, mais également des institutions de protection de la jeunesse, est centrale. Toutes ces réactions peuvent se conjuguer pour créer un sentiment de trahison et de perte $d^{\prime}$ 'une figure importante.

La littérature empirique sur la dépression confirme la théorie psychanalytique de la perte d'objet comme un facteur prédisposant à la dépression (Peters, 1984). Ici le mot «perte» peut signifier la mort d'un parent, une séparation prolongée, mais aussi la perte d'un parent sur le plan de la relation. La tristesse et les éléments de dépression chez les victimes peuvent s'expli-quer par la réaction de l'enfant à la perte, par la trahison, d'une figure importante.

Le besoin de retrouver la confiance et la sécurité perdues amène parfois l'enfant à démontrer des comportements de dépendance excessive (Finkelhor, 1986). Par ailleurs, la victime peut développer, en réaction à la trahison, des comportements d'hostilité, de colère et d'isolement (Finkelhor, 1986).

Parvenues à l'âge adulte, les victimes manifestent des sentiments $\mathrm{d}^{\prime}$ isolement ainsi que des problèmes relationnels, voire conjugaux. Ainsi, Meiselman (1978) confirme que la victime continue d'éprouver des sentiments négatifs à l'égard de sa mère. Meiselman (1978) et Russell (1986) parlent de sentiments négatifs à l'égard de l'abuseur, tandis que Briere (1984), 
Meiselman (1978), Herman (1978) et Gorcey, Santiago et McCall-Perez (1986), avec des populations cliniques, ainsi que Courtois (1979) et Russell (1986), avec des populations non cliniques, maintiennent que les victimes vivent des sentiments de peur, de colère ou de méfiance à l'égard des hommes.

Enfin, Goodwin, McCarthy et DiVasto (1981) établissent une corrélation entre le fait d'avoir été victime d'abus étant enfant et celui de vivre avec un homme qui abuse de ses propres enfants. Plusieurs recherches relient enfin les abus sexuels à une revictimisation subséquente (Russell, 1986; Bagley et MacDonald, 1984 ; Hartman et al., 1987 ; Gorcey et al., 1986 ; Briere, 1984 ; Jehu, 1989; Gorcey et al., 1986 ; Hartman, Finn et Leon, 1987).

\section{L'impuissance}

L'impuissance est la conséquence du processus par lequel la volonté, les désirs et le sens d'efficacité de l'enfant sont transgressés. Le sentiment que l'espace corporel est envahi de façon répétée, sans que la victime y consente, est central dans les abus sexuels. Le sentiment d'impuissance augmente quand l'enfant tente de contrer l'abus sans succès.

Les expériences d'abus qui impliquent de la violence physique ou des menaces de violence produisent un sentiment d'impuissance plus aigu. L'enfant expérimente alors une peur de se faire blesser ou de se faire tuer s'il n'accepte pas de participer à l'abus. Ici, la victime n'a pas simplement le sentiment d'être trahie, mais ressent plutôt que sa vie est en danger.

Anderson et al. (1981) ont décelé des séquelles psychosociales intériorisées (problèmes de sommeil et d'alimentation, peurs et phobies, dépression) et des séquelles extériorisées (problèmes scolaires et fugues). D'autres auteurs (Peters, 1976) confirment l'existence de problèmes de sommeil et d'alimentation.

Les événements qui suivent le dévoilement de l'abus peuvent également contribuer à exacerber le sentiment d'impuissance, quand les réactions de l'entourage immédiat, de la police et des services sociaux ne tiennent pas compte des besoins de la victime.

Problèmes psychosomatiques, d'alimentation, d'emploi, délinquance, voici certains problèmes ressentis à l'âge adulte que nous pouvons relier à I'impuissance (Van Buskirk, 1983; Vander Mey, 1982 ; Alter-Reid, 1986). Également à l'âge adulte, l'ex-victime aura tendance à revivre des victimisations.

Enfin, les victimes d'abus sexuels qui ont vécu beaucoup d'impuissance au cours de l'abus peuvent manifester des symptômes comme de la peur, des problèmes d'évitement et de dissociation (Edwards et Donaldson, 1989; Briere, 1989 ; Courtois, 1989). 


\section{Critiques}

Comme nous venons de le voir, la théorie des quatre dynamiques traumatisantes a pour effet de permettre de saisir les conséquences des abus sexuels dans leur ensemble plutôt que comme des éléments isolés les uns des autres. En ce sens, son utilisation est intéressante tant en intervention qu'en recherche.

Toutefois, le modèle a ses lacunes que plusieurs théories existantes peuvent combler. Le processus des quatre dynamiques traumatisantes n'explique pas pourquoi les victimes ont tendance à se sentir responsables de l'abus. Les théories sur l'impuissance acquise (Vallerand et Bouffard, 1985) nous permettent de mieux saisir le phénomène d'auto-responsabilisation (selfblame). Ainsi, selon les auteurs, nos interactions avec l'environnement social ne sont pas contrôlées par des faits objectifs, mais bien par notre perception de ces faits. Les victimes en viennent ainsi à se sentir responsables d'un abus qu'elles ont en fait subi (Hoagwood,1990). Par contre, les auteurs n'arrivent pas à expliquer pourquoi des déformations cognitives se produisent. Autrement dit, pourquoi et comment les femmes en viennent-elles à se sentir responsables de l'abus? Nous devons chercher une réponse ailleurs. Le modèle de Finkelhor et Browne n'arrive pas non plus à expliquer les causes des abus sexuels. Il ne tient pas compte du fait que les victimes sont très majoritairement de sexe féminin. Les auteurs n'opèrent pas de jonction avec des facteurs sociaux qui viendraient agir pour renforcer ce processus. C'est ici que la théorie féministe nous apporte un éclairage beaucoup plus cohérent sur ces deux phénomènes.

La théorie féministe redéfinit l'inceste et les abus sexuels (Courtois, 1988 ; Meiselman, 1990 ; Herman, 1987 ; Russell, 1986 ; Joy, 1987) comme un désir de pouvoir et de dégradation des femmes par les hommes, les hommes étant conditionnés dans des rôles de pouvoir et de domination à l'égard des femmes. La sexualité n'est qu'un autre domaine où les hommes revendiquent ce pouvoir. La formation de symptômes est perçue, d'une part, comme la conséquence de cette domination et, d'autre part, comme un mécanisme qui permet à la victime de survivre à l'inceste et à ses conséquences (Courtois, 1988 ; Brickman, 1984 ; Dominelli, 1989).

Quant à la question d'auto-responsabilisation, c'est du côté de la socialisation des femmes que les féministes cherchent la réponse. Ce processus à travers lequel les femmes apprennent leur rôle de victime les amène également à se sentir responsables des abus qu'elles subissent. Pour les féministes, les femmes ne désirent pas plus être «abusées » qu'elle ne désirent être violées, et les féministes ont élargi à toutes les formes d'agressions sexuelles leur critique de la théorie psychanalytique du désir du viol (Brownmiller, 1975). 
L'avantage de cette analyse se trouve dans le fait qu'elle ne particularise pas le problème. D'un problème individuel, l'abus devient un problème social. De plus, la dénonciation des abus sous toutes leurs formes place le problème sur un autre terrain qui débouche inéluctablement sur sa prévention. En effet, dénoncer la situation, permet, à long terme, d'empêcher la reproduction, de génération en génération, de toutes les formes d'abus et de violence.

\section{CONCLUSION}

La théorie des quatre dynamiques traumatisantes nous décrit le processus qui amène le développement des conséquences à court et à long terme des abus sexuels. Elle a l'avantage d'organiser la liste des innombrables conséquences en un tout cohérent.

Par contre, ce modèle néglige certains aspects des abus dont nous devons tenir compte tant sur le plan de l'intervention que de la recherche. Certaines théories, comme les théories de l'attribution, de l'impuissance acquise et la théorie féministe, nous amènent un éclairage essentiel à la compréhension des conséquences de l'inceste et des abus sexuels.

\section{Références bibliographiques}

ALteR-Reid, K. et al. (1986). "Sexual abuse of children: a review of the empirical findings », Clinical Psychology Review, vol. 6, n 4 : 249-266.

BAGLEY, C. et R. RAMSEY (1985). «Sexual abuse in childhood: psychosocial outcomes and implications for social work practice », Journal of Social Work and Human Sexuality, vol. $4: 33-47$.

BAGLEY, C. et M. MCDONALD (1984). «Adult mental health sequels of child sexual abuse, physical abuse and neglect in maternally separated children », Canadian Journal of Community Mental Health, vol. 3, n 1: 15-26.

BECKER, J.V. (1984). "Sexual problems of sexual survivors», Women and Health, vol. 9, no $4: 5-20$.

BRIERE, J. et M. RUNTZ (1987). «Post sexual abuse trauma », Journal of Interpersonal Violence, vol. 2, $\mathrm{n}^{\circ}$ 4: 367-379.

BRICKMAN, J. (1984). « Feminist, nonsexist, and traditional models of therapy: implications for working with incest», Women and Therapy, vol. 3, n 1:49-67.

BROWNMILLER, S. (1975). Against our Will: Men, Women and Rape. New York: Simon and Shuster.

CONTE, J. (1985). "The effects of sexual abuse on children: a critique and suggestions for future research », Victimology: an International Journal, vol. 10, $\mathrm{n}^{\text {os }} 1-4$ : 110-130. 
COOPERSMith, S. (1967). The Antecedents of Self-Esteem. San Francisco: Freeman and Co.

COURTOIS, C. (1984). "The Incest Experience and Its Aftermath», Victimology, vol. 4, no $4: 337-347$.

COURTOIS, C. (1988). Healing the Incest Wound, New York: W. Norton \& Company.

DE YOUNG, M. (1982). «Self-injurious behaviour in incest victims : a research note», Child Welfare, vol. $61: 577-584$.

DOMINELLI, D. (1989). «Betrayal of trust: a feminist analysis of power relationships in incest abuse and its relevance for social work practice», British Journal of Social Work, vol. 19: 291-307.

EARLS, C. et H. DAVID (1990). «Expériences familiales et sexuelles précoces des hommes et des femmes prostitués», Santé mentale au Canada, déc. : 7-12.

EDWARDS, P. et M.A. DONALDSON (1989). «Assessment of symptoms in adult survivors of incest: a factor analytic study of responses to childhood incest questionnaire », Child Abuse and Neglect, vol. 13: 101-110.

FINKELHOR, D. (1979). Sexually Victimized Children. New York: Macmillan Free Press.

FINKELHOR, D. et A. BROWNE (1985). «The traumatic impact of child sexual abuse: a conceptualization, American Journal of Orthopsychiatry, vol. 55, $\mathrm{n}^{\circ} 4$ : 530-541.

FINKELHOR, D. et al. (1986). A Sourcebook on Child Sexual Abuse. Beverly Hills: Sage.

FINKELHOR, F. (1984). Child Sexual Abuse: New Theory and Research. New York: Macmillan.

GOODWIN, J., T. MCCARTHY et P. DIVASTO (1981). «Prior incest in mothers of abused children », Child Abuse and Neglect, vol. 5 : 87-95.

GORCEY, M., J.M. SANTIAGO et F. MCCALL-Perez (1986). "Psychological consequences for women sexually abused in childhood », Social Psychiatry, vol. 21 : 129-133.

HARTMAN, M., S. FINN et G. LEON (1987) «Sexual-abuse experiences in a clinical population: comparisons of familial and non-familial abuse», Psychotherapy, vol. 24, $\mathrm{n}^{\circ} 2: 154-159$.

HERMAN, J. (1981). Father-daughter Incest. Cambridge: Harvard University Press.

HERMAN, J., D. RUSSELL et K. TROCKI (1986). «Long-term effects of incestuous abuse in childhood», American Journal of Psychiatry, vol. 143, n 10: 1293-1296.

HERMAN, J. ET E. SCHATZOW (1987). "Recovery and verification of memories of childhood sexual trauma ", Psychoanalytic Psychology, vol. 4, n 1:1-14.

HOAGWOOD, K. (1990). «Blame and adjustment among women sexually abused as children », Women and Therapy, vol. 9, n 4: 89-110.

JEHU, D. (1989). "Mood disturbances among women clients sexually abused in childhood », Journal of Interpersonal Violence, vol. 4, $\mathrm{n}^{\circ} 2$ : 164-184.

JOY, S. (1987). «Retrospective presentation of incest: treatment strategies for use with adult women », Journal of Counseling and Development, vol. 65, $\mathrm{n}^{\circ} 6$.

MeISELMAN, K. (1978). Incest. San-Francisco: Jossey-Bass.

PETERS, S.D. (1984). The relationship between childhood sexual victimization and depression among Afro-American and white women. Los Angeles : University of California. 
RUSSELL, D. (1984). Sexual exploitation, rape, child sexual abuse and workplace harassment. Beverly Hills : Sage.

RUSSELL, D.H. (1986). The secret trauma, incest in the lives of girls and women. New York: Basic Books.

SEDNEY, M.A. et B. BROOKS (1984). «Factors associated with a history of childhood sexual experience in a nonclinical female population », Journal of the American Academy of Child Psychiatry, vol. 23, $\mathrm{n}^{\circ} 2: 215-218$.

TSAI, M. et N.N. WAGNER (1978). "Therapy groups for women sexually molested as children », Archives of Sexual Behavior, vol. 7, n $5: 417-427$.

VALLERAND, R.J. et L. BOUfFARD (1985). "Concepts et théories en attribution », Revue québécoise de psychologie, vol. 6, $\mathrm{n}^{\circ} 2$ : 46-65.

VAN BUSKIRK, S. et C. COLE (1983). "Characteristics of eight women seeking therapy for the effects of incest "Psychotherapy: Theory, Research and Practice, vol. 20, : 503-514.

VANDER MEY, B. et R. NEFF (1982). "Adult-child incest: a review of research and treatment », Adolescence, vol. 7, n $68: 713-735$.

WHEELER, D. et E. WALTON (1987). «Personality disturbances of adult incest victims », Social Casework, vol. 68, n 9: 597-602. 